\title{
Tunable time-reversal cavity for high-pressure ultrasonic pulses generation: A tradeoff between transmission and time compression
}

Bastien Arnal, Mathieu Pernot, Mathias Fink, and Mickael Tanter

Citation: Appl. Phys. Lett. 101, 064104 (2012);

View online: https://doi.org/10.1063/1.4742930

View Table of Contents: http://aip.scitation.org/toc/apl/101/6

Published by the American Institute of Physics

\section{Articles you may be interested in}

Time reversal transfer: Exploring the robustness of time reversed acoustics in media with geometry perturbations The Journal of the Acoustical Society of America 138, EL49 (2015); 10.1121/1.4922623

Two-dimensional virtual array for ultrasonic nondestructive evaluation using a time-reversal chaotic cavity The Journal of the Acoustical Society of America 130, 2720 (2011); 10.1121/1.3643828

\section{Scilight} Sharp, quick summaries illuminating the latest physics research

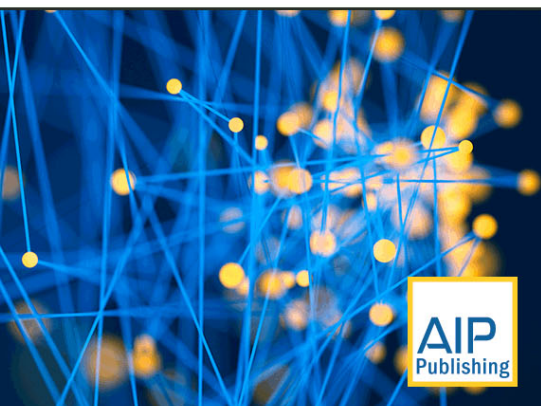




\title{
Tunable time-reversal cavity for high-pressure ultrasonic pulses generation: A tradeoff between transmission and time compression
}

\author{
Bastien Arnal, Mathieu Pernot, Mathias Fink, and Mickael Tanter \\ Institut Langevin, ESPCI ParisTech, CNRS UMR 7587, INSERM U979, Université Paris Diderot, \\ Paris 75005 , France
}

(Received 16 May 2012; accepted 24 July 2012; published online 7 August 2012)

\begin{abstract}
This Letter presents a time reversal cavity that has both a high reverberation time and a good transmission factor. A multiple scattering medium has been embedded inside a fluid-filled reverberating cavity. This allows creating smart ultrasonic sources able to generate very high pressure pulses at the focus outside the cavity with large steering capabilities. Experiments demonstrate a $25 \mathrm{~dB}$ gain in pressure at the focus. This concept will enable us to convert conventional ultrasonic imaging probes driven by low power electronics into high power probes for therapeutic applications requiring high pressure focused pulses, such as histotripsy or lithotripsy. (C) 2012 American Institute of Physics. [http://dx.doi.org/10.1063/1.4742930]
\end{abstract}

In biomedical ultrasound applications, conventional transducers are grouped in multi-element arrays to create dynamic electronic focusing of broadband signals. For therapeutic applications, high power transducers $(\sim 100-500$ elements, large diameter $\sim[3 \lambda-10 \lambda]$ ) are usually placed on a spherical shell, allowing to focus at the center of the sphere and around the center, thanks to electronic steering of the beam. Nevertheless, the beam steering is geometrically limited to a small region around the geometrical focus because of directivity of the large elements. ${ }^{1,2}$ Time-reversal (TR) focusing into a reverberating medium is an adaptive focusing method that takes advantage of disordered media to provide dynamic spatio-temporal focusing within a large region of interest using a very limited number of transmit elements. The use of a time-reversal cavity (TRC) allows creating many virtual acoustic sources, thanks to the multiple reverberations inside the cavity. In 1997, an experiment of TR focusing (TRF) in a waveguide showed the benefits of a bounded medium to sharpen the focal spot. ${ }^{3}$ Usually, TRF is comprised of two steps. A short broadband signal is emitted from a source and recorded by the array. These signals are then reversed in time and reemitted from the array. By exploiting the TR invariance and spatial reciprocity of the wave equation, the signals refocus on the initial source, both in space and time. Thus, TR provides an elegant way to apply the complex temporal signals on each element of the TRC in order to create a multitude of virtual elements and refocus optimally at the desired location. Instead of using a large spatial distribution of elements, most of the transmit wave complexity is transferred into the time dimension.

Another advantage of TRC consists in strongly amplifying the pulse amplitude at the focus by recompressing very long transmitted signals $(\sim 1 \mathrm{~ms})$ into very short impulses at the focus $(\sim 1 \mu \mathrm{s})$. Montaldo et al. used a solid metallic cavity $^{4}$ in order to focus an ultrasonic pulse into water. This closed cavity strongly limited the transmitted energy into the target medium. The initial longitudinal wave was shown to be gradually converted to shear waves until the shear being preponderant. However, the abrupt impedance mismatch at the interface, which provided the high quality factor (Q) of the resonator, limited the pulse transmission $(\mathrm{T} \sim 0.1)$ into the target medium. Even though this metallic cavity had low leakage (see Fig. 1(a)), it was found possible to apply this concept to $3 \mathrm{D}$ ultrasonic imaging with acceptable performances. 5,6

In contrast, Sarvazyan et al. used a water filled reverberating cavity made of a plastic bottle surrounded by air and opened at one extremity. ${ }^{7}$ This device was capable of

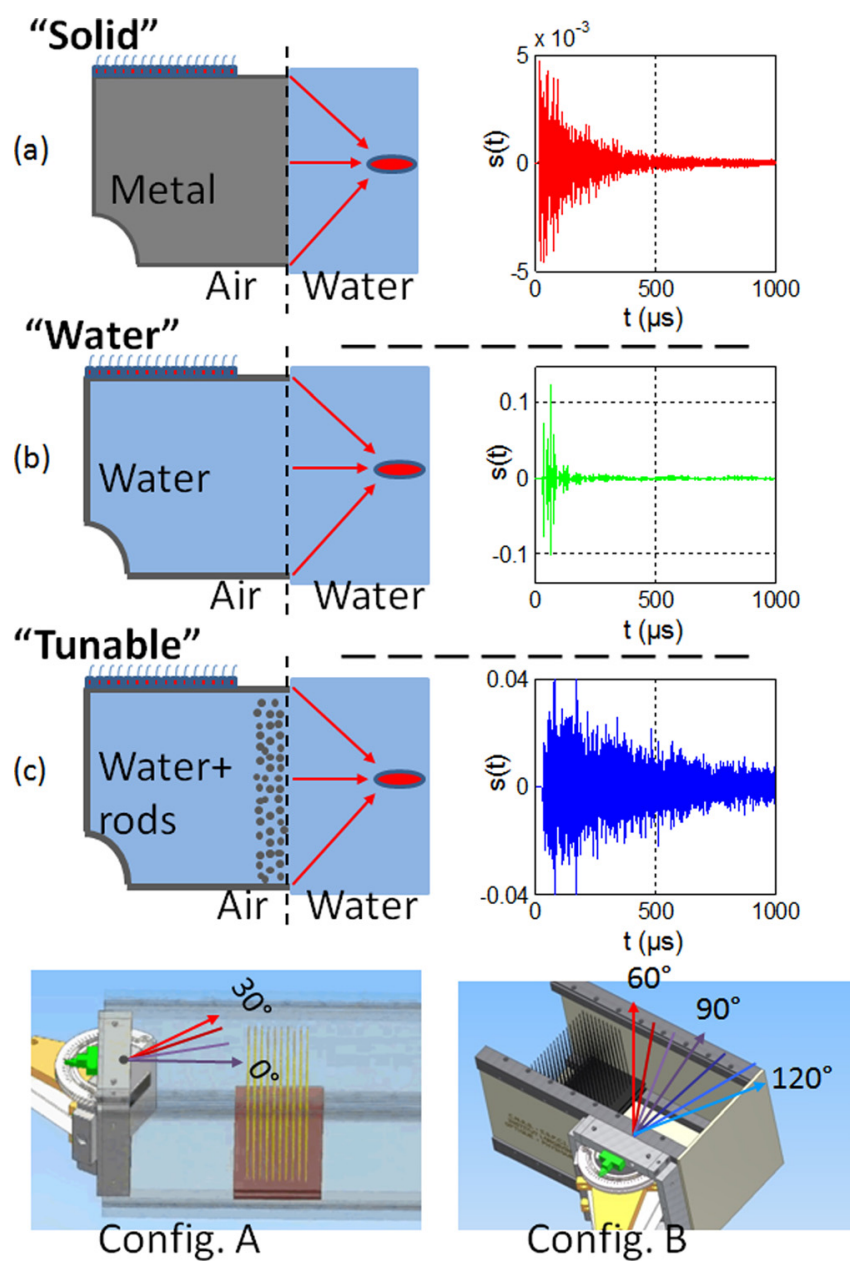

FIG. 1. (a) Solid TRC, (b) water TRC, and (c) tunable TRC. From left to right: Concept scheme of the TRC, a typical signal obtained by a FDTD simulation. Below (c): Our experimental realization in 2 configurations A and B (see further). 
generating one focus or several foci and of steering them electronically at a considerable distance off the axis of the focusing system without any grating lobes or secondary intensity maxima. Nevertheless, this opened cavity did not show a strong amplitude level enhancement at the focus since this device acted as a resonator with a low quality factor and a strong leakage (see Fig. 1(b)).

To overcome the tradeoff, we propose here an approach based on a leaky cavity (liquid filled cavity) combined with a high-order multiple scattering medium (MSM) to improve the quality factor (see Fig. 1(c)). Contrary to previous approaches, our technique takes advantage of both a long reverberation time and a good transmission coefficient of the device. Moreover, these two parameters can be tuned by changing the spatial distribution of scatterers. An MSM made of steel rods can temporally disperse the signal on hundreds of periods. It is well known that a high-order MSM leads to an improvement of the signal-to-noise ratio (SNR) and spatial resolution at the focus. ${ }^{8}$ One way to increase further the amplitude of the TR pulse at the focus is to use 1-bit $\mathrm{TR}^{8}{ }^{8}$ It consists in applying a one-bit quantization on the reemitted signals $(+1$ if $\mathrm{s}(\mathrm{t})>0,-1$ if $\mathrm{s}(\mathrm{t})<0)$. Derode et $_{\text {al. }}{ }^{8}$ have shown that 1-bit TR through a 2D MSM could amplify the compressed pulse by $12 \mathrm{~dB}$, while the temporal and spatial resolutions remained. It means that TR-focusing does not need the amplitude to be efficient and 1-bit TR enables emitting more energy. Another advantage of 1-bit TR is to use simple and low cost electronic pulsers. However, in Derode et al., ${ }^{8}$ ultrasonic waves scattered in every direction were lost so that they did not contribute to the focusing. This loss could have been avoided if the scattering medium had been placed inside a TRC. In this case, the MSM would have been sonicated several times by different modes of the cavity. Hence, this concept leads to a tunable TRC where the MSM adapts the cavity leakage as desired. As an illustration, we performed three 2D finite differences time domain (FDTD) acoustic simulations of our proposed cavity as well as the two existing types of cavities (solid and water). Results in terms of relative pressure obtained at the focus are given in Table I.

The tunable TRC leads to an amplification of $\times 15$ compared to the solid cavity used in 1-bit TR. To justify this result, the shot noise model proposed by Derode et al ${ }^{8,9}$ was used. The random signals can be described with a variance $\sigma(\mathrm{t})$, representing the envelope of the signal $\mathrm{s}(\mathrm{t})$. Assuming a simple case where the signal has an exponential decay, we can write $\sigma(\mathrm{t})$, the expected value of the TR pulse $\mathrm{r}(\mathrm{t})$, and the 1 bit-TR pulse $\mathrm{u}(\mathrm{t})$ as follows:

$$
\begin{aligned}
\sigma(t) & =M \cdot u(t) e^{-\alpha t}, \\
E\{r(t)\} & =\frac{1}{M} \rho(t) \int \sigma^{2}(\theta) d \theta=\frac{M}{2 \alpha} \rho(t), \\
E\{u(t)\} & =\sqrt{\frac{2}{\pi}} \rho(t) \int \sigma(\theta) d \theta=\sqrt{\frac{2}{\pi}} \frac{M}{\alpha} \rho(t),
\end{aligned}
$$

where $M$ is the maximum amplitude of the signal $s(t)$ (impulse response), $u(t)$ is the Heavyside function, $\alpha$ is the decay constant of the signal $\left(\mathrm{s}^{-1}\right), \rho$ is the correlation coefficient between $\mathrm{s}\left(\mathrm{t}_{1}\right)$ and $\mathrm{s}\left(\mathrm{t}_{2}\right)$, and the symbol $\mathrm{E}\{\}$ denotes an
TABLE I. Normalized pressure at the focus obtained in FDTD simulations (same transmitted acoustic intensity for each configuration). Left column: time-reversal; right column: 1-bit TR.

\begin{tabular}{lcc}
\hline \hline & $\mathrm{P} / \mathrm{P}_{0}(\mathrm{TR})$ & $\mathrm{P} / \mathrm{P}_{0}(1$ bit-TR $)$ \\
\hline Solid cavity & 1.00 & 6.44 \\
Water cavity & 5.03 & 28.39 \\
Tunable cavity & 22.65 & 96.47 \\
\hline \hline
\end{tabular}

ensemble average. The TR-pressure is then both dependent of the time-compression denoted by the decay time $1 / \alpha$ as well as the transmission of the cavity, which is proportional to $\mathrm{M}$. In the current example, the main improvement from the solid to the tunable TRC is a higher transmission (M $8 \times$ larger).

In our design, we need an MSM with good broadband properties and a cavity made of a good reflector. We will show how to choose the MSM in a 2D configuration. Then, we investigate numerically and experimentally the optimal density of scatterers and the optimal length of scattering medium. The optimal transmission factor of the TRC results from a tradeoff between the effective transmission coefficient and the gain provided by multiple paths. Hence, our device is a tunable TRC. In addition to the beam-steering abilities, our main goal was to obtain the maximum pressure amplitude. The pressure measurements were performed using a needle hydrophone (HGL 200, ONDA Corp, Sunnyvale, USA) in the linear acoustic regime $(\mathrm{P}<2 \mathrm{MPa})$.

A single scatterer is characterized by its scattering cross section $\sigma$ and its directivity expressed as the mean cosine $\langle\cos \rangle$. To maximize temporal dispersion, the scattering mean free path $l_{e}$ has to be minimized, while the transport mean free path $l^{*}$ has to be maximized to ensure a good transmission. As $l_{e}=1 /(n \sigma)$ and $l^{*}=l e /(1-\langle\cos \rangle),{ }^{10}$ where $n$ is the surface density of scatterers, the scatterer must have a maximum $\sigma$ and a minimum $\langle\cos \rangle$ at the working frequency. As we manipulate broadband signals, no resonance has to be present within the bandwidth of our probe. Using an analytic calculation of $\sigma(f)$ and $\langle\cos \rangle(f)$ for various steel rod diameters, ${ }^{11}$ we found that there was a flat frequency domain $([0.75-2.75] \mathrm{MHz})$ on the curves $\sigma(f)$ and $\langle\cos \rangle(f)$ with maxima at $1.7 \mathrm{MHz}$ for a steel rod diameter of $0.8 \mathrm{~mm}$ $\left(\sigma_{\max }=1.3 \mathrm{~mm},\langle\cos \rangle=0.2\right)$ (see Figs. 2(a) and 2(b)). Next, these rods were placed randomly in a surface of $9 \mathrm{~cm} \times \mathrm{L}$, where $\mathrm{L}$ is the width of the multiple scattering, see Fig. 1(c). Then, the influence of the rod density was investigated in simulations using a fixed width $(\mathrm{L}=3 \mathrm{~cm})$.

FDTD acoustic simulations in 2D were performed using different MSM configurations. A linear diagnostic probe (pitch: $0.7 \mathrm{~mm}$, width: $9 \mathrm{~cm}$ ) was placed on one side of the MSM $(\mathrm{L}=3 \mathrm{~cm})$. A short pulse (central frequency $1 \mathrm{MHz}$, $3 \mu \mathrm{s})$ was emitted from the target point, and the probe recorded the long signal $(\sim 300 \mu \mathrm{s})$, which was re-emitted after the TR operation. To estimate the pressure obtained using 1-bit TR, the classic TR pressure was multiplied by the theoretical gain corresponding to the shot noise model. ${ }^{8}$ This ratio typically ranged from 3 to 5 .

From Fig. 2(b), the best density appeared to be between 8 and $10 \mathrm{rods} / \mathrm{cm}^{2}$. The decrease of the peak pressure at 
(a)

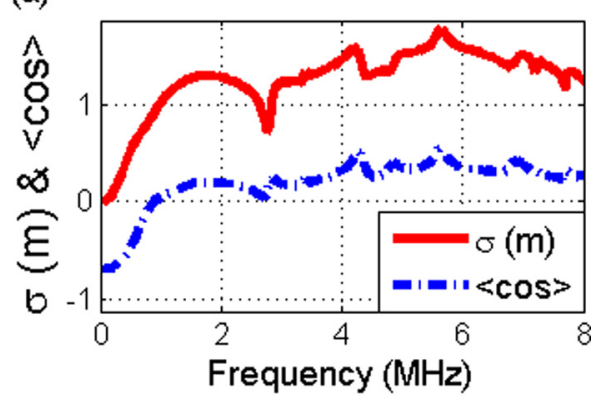

(b)

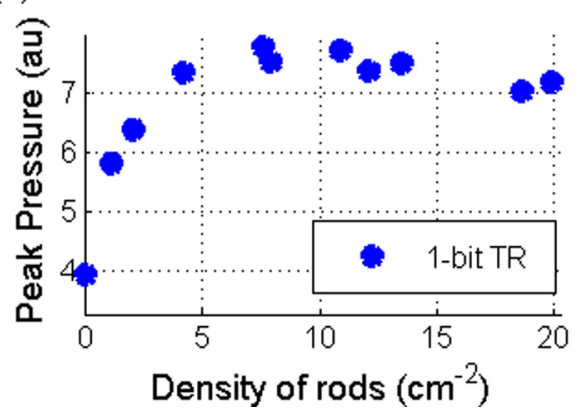

FIG. 2. (a) Theoretical scattering cross section and mean cosine of a $0.8 \mathrm{~mm}$ diameter steel rod. (b) FDTD simulation. A linear probe is simulated to focus at $3 \mathrm{~cm}$ through the rods. Effect of the density of rods on the pressure value using classic TR and the 1-bit TR deducted value using the theoretical gain.

higher densities can be explained by the loss due to the scattered energy at the absorbing boundaries. The optimum density results from a tradeoff between time-compression and losses. These simulations allow finding a good range of scatterer density to perform experiments with the addition of the cavity.

We built a $2 \mathrm{D}$-waveguide made of reflecting duralumin plates filled with water $(\operatorname{dim} 14 \times 14 \times 25 \mathrm{~cm})$. A diagnostic probe $(1.5 \mathrm{MHz}, 128$ elements, $-3 \mathrm{~dB}$ bandwidth: $80 \%$, Vermon, Tours, France) connected to a fully programmable multichannel electronics was fixed onto a rotating holder allowing different spatial excitations. We adopted two distinct configurations shown on Fig. 1(c): (A) The probe was placed along the length of the cavity and steered from $-30^{\circ}$ to $30^{\circ}$; (B) The probe was placed transversally and steered from $60^{\circ}$ to $120^{\circ}$. A steel rods medium $(0.8 \mathrm{~mm}$ diameter $)$ was placed inside the cavity. Here, we used a fixed rod density of 10 rods $/ \mathrm{cm}^{2}$ as found in simulations, and the width L of the medium was changed from $0 \mathrm{~cm}$ to $6 \mathrm{~cm}$ (see Fig. 3).

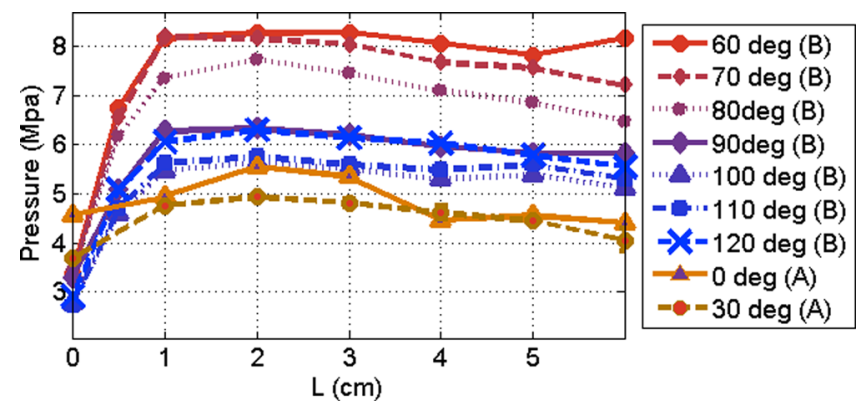

FIG. 3. Effect of the width of the scattering medium in the configurations (A) and (B) for several angulations of the probe.
In configuration $\mathrm{A}$, the pressure was maximized for a scattering medium of $2 \mathrm{~cm}$. The observation that the pressure was found to decrease in thicker media indicated some losses. To analyze if the losses were due to the absorption of the backscattered wave on the transducers, the configuration $\mathrm{B}$ was investigated with the transducer positioned on the side of the cavity.

In configuration $\mathrm{B}$, a higher pressure was obtained from $1 \mathrm{~cm}$ thick scattering medium, and the same pressure was observed for thicker medium. This demonstrates that this configuration reduced greatly the losses of the backscattered waves. The sonication angle had a significant effect in this configuration. From $90^{\circ}$ to $120^{\circ}$, the beams had to bounce on the reflector, and a part of energy was absorbed back by the probe. In contrast, the angulations between $60^{\circ}$ and $80^{\circ}$ gave higher pressures since the beams were directed toward the rods, and the backscattered energy reflected on the back plate and re-injected to the rods forest. Less energy was absorbed on the probe. For a given angle, the increase of $\mathrm{L}$ did not lead to a significant decrease of the gain as the absorption coefficient in water remained negligible. At $60^{\circ}$ with $\mathrm{L}=3 \mathrm{~cm}$, the gain in pressure compared to the conventional focusing with the same probe was $\times 6.4$, given the very large width of the focal spot in elevation (DY $=20 \mathrm{~mm}$ ) because of diffraction. We first improved focusing in elevation using a plane-convex PDMS lens (DY $=4 \mathrm{~mm}$ ), leading to an enhancement to $\times 12.3$. Finally, we replaced the duralumin plates that had a reflection factor $(\mathrm{R}=0.8)$ by a water-air interface $(R=-0.99)$. These walls of the cavity were made of a frame holding a tight aluminum foil with a $2 \mathrm{~mm}$ trapped air layer on the other side. This perfect reflector further improved the gain up to $\times 17$ ( $25 \mathrm{~dB})$. Note that these results were obtained in the linear acoustic regime $(\mathrm{P}<2 \mathrm{MPa})$. In the field of TR microwaves, a comparable gain has been obtained $(18 \mathrm{~dB})$ by Davy et al. ${ }^{12}$

Configuration A was considered in terms of beam steering capabilities. TR-focusing was achieved at several lateral positions. The directivity pattern of the probe alone was measured at $10 \mathrm{~cm}$ ("water" on Fig. 4(a)). Then, the probe
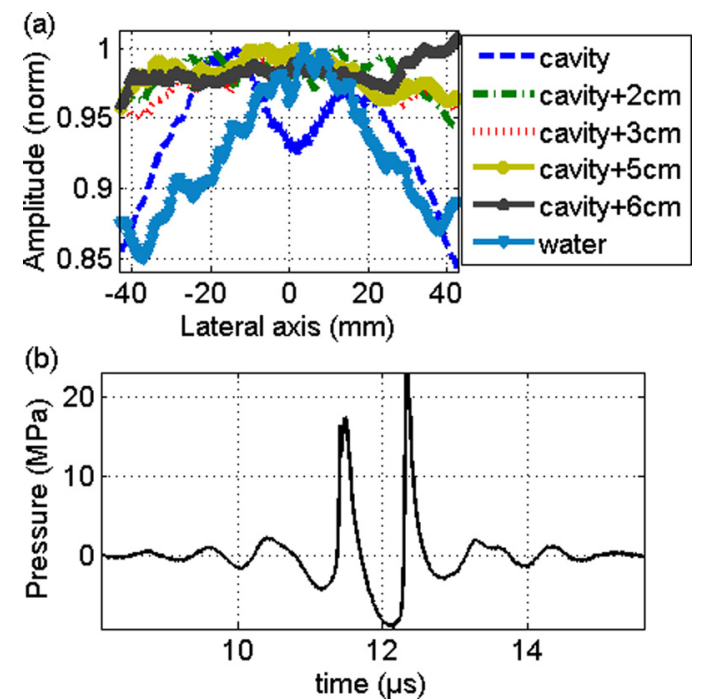

FIG. 4. (a) Beamsteering diagram in configuration A. (b) Shock wave obtained with the optimal TRC at maximum output voltage $\left(\mathrm{P}_{+}=23.3 \mathrm{MPa}\right.$, $\left.\mathrm{P}_{-}=-9.0 \mathrm{MPa}\right)$. 
was placed inside the cavity with a $0^{\circ}$ angulation. The curve ("Cavity") showed that the directivity pattern of the cavity is governed by the reflections. Finally, the addition of the rod achieved a very flat area of beam steering over the whole aperture, even with $\mathrm{L}=2 \mathrm{~cm}$ (Fig. 4(a)).

Finally, the high power capability of the TRC was assessed experimentally. In the first step, a hydrophone was used to record the signal. Then, the hydrophone was replaced by a mylar film whose displacements were measured using an optical interferometer withstanding very high pressure broadband signals. ${ }^{13}$ As shown in Fig. 4(b), the ultrasonic wave was strongly distorted at the focus, and a shockwave was observed at $12.3 \mu \mathrm{s}$. The bandwidth of the diagnostic probe used $(80 \%)$ allowed us to generate a wideband shock wave. Same pressure level was reached with a 3.5 cycles signal. With our current system, the peak positive pressure was $23.3 \mathrm{MPa}$, whereas the peak negative pressure was $-9.0 \mathrm{MPa}$. In contrast, conventional focusing gave $1.3 \mathrm{MPa}$ at the same distance and voltage. This confirmed the important gain found in the linear regime. The peak negative pressure was limited by the high nonlinear propagation in $2 \mathrm{D}$ focusing. In the elevation direction, the quasi-plane wave led to strong nonlinear effects. In a 3D focusing system, a better antenna gain would allow to reach a higher peak negative pressure. Nevertheless, we assume that the wave propagated linearly inside our cavity, thanks to the polarity inversion at each reflection on the water-air walls, which reduced the nonlinear effects. ${ }^{14}$ Nonlinear propagation may have occurred mainly outside the cavity, where the pressure was significantly high. This nonlinearity will be investigated in a further study.

In summary, we performed simulations and experiments to demonstrate the feasibility of tuning the leakage of a TRC. The optimal mean free paths were estimated: $l e \simeq 0.26 \mathrm{~L}$ and $l^{*} \simeq 0.32 \mathrm{~L}$. The ratio between the amplitudes of the ballistic waves into the configurations with or without the rods gave a mean transmission factor of $\mathrm{T}=0.3$. The multiple scattering medium embedded into the fluid-filled TRC provided such a transmission factor ranging between the transmission of a solid cavity $(\mathrm{T}=0.1)$ and an opened one $(\mathrm{T}=1)$. We converted a diagnostic probe with low voltage electronics into a high power $(25 \mathrm{~dB}$ pressure gain at the focus) therapeutic transducer with high steering capabilities for lithotripsy or histotripsy applications.

The authors thank Abdelhak Souilah for the design, manufacture, and improvements of the system.

${ }^{1}$ L. R. Gavrilov and J. W. Hand, IEEE Trans. Ultrason. Ferroelectr. Freq. Control 47, 125 (2000).

${ }^{2}$ M. Pernot, J.-F. Aubry, M. Tanter, J.-L. Thomas, and M. Fink, Phys. Med. Biol. 48, 2577 (2003).

${ }^{3}$ P. Roux, B. Roman, and M. Fink, Appl. Phys. Lett. 70, 1811 (1997).

${ }^{4}$ G. Montaldo, P. Roux, A. Derode, C. Negreira, and M. Fink, J. Acoust. Soc. Am. 110, 2849 (2001).

${ }^{5}$ G. Montaldo, D. Palacio, M. Tanter, and M. Fink, Appl. Phys. Lett. 84, 3879 (2004).

${ }^{6} \mathrm{G}$. Montaldo, D. Palacio, M. Tanter, and M. Fink, IEEE Trans. Ultrason. Ferroelectr. Freq. Control 52, 1489 (2005).

${ }^{7}$ A. P. Sarvazyan, L. Fillinger, and L. R. Gavrilov, Acoust. Phys. 55, 630 (2009).

${ }^{8}$ A. Derode, A. Tourin, and M. Fink, J. Appl. Phys. 85, 6343 (1999).

${ }^{9}$ K. J. Haworth, J. B. Fowlkes, P. L. Carson, and O. D. Kripfgans, J. Acoust. Soc. Am. 125, 3129 (2009).

${ }^{10}$ A. Ishimaru, Wave Propagation and Scattering in Random Media (John Wiley and Sons, 1999).

${ }^{11}$ L. Flax, G. C. Gaunaurd, and H. Uberall, Physical Acoustics 15, 191-294 (1981).

${ }^{12}$ M. Davy, J. de Rosny, J.-C. Joly, and M. Fink, C. R. Phys. 11, 37 (2010).

${ }^{13}$ D. Royer, N. Dubois, and M. Fink, Appl. Phys. Lett. 61, 153 (1992).

${ }^{14}$ M. Tanter, J. L. Thomas, F. Coulouvrat, and M. Fink, Phys. Rev. E 64, 016602 (2001). 